\title{
The Mathematical Modeling and software Simulation for Ship Oil Purifier System
}

\author{
Ning Zhang \\ School of Fore ign languages \\ Dalian Maritime University \\ Dalian, China
}

\begin{abstract}
There are lots of limitations in ship oil purifier practical training system. Most of the simulation system is given priority to hardware simulation and it isn't convenient to use. For this purpose, it's necessary to design a software simulation platform to help students to master the application of oil purifier system. The article uses the oil purifier system in the COSCO PACIFIC thousands of container ship as the simulation object. By using VC++ technology to simulate the operation of the ship oil purifier function, the system established the oil purifier simulation system mathematical model, realized the control unit EPC50 logic control and designed the system interface. Through operating the proposed simulation system, it shows that the mathematical model is accurate and the system can comprehensively and realistically reflect the working condition and the actual dynamic-process of oil purifier system.
\end{abstract}

Keywords-ship oil purifier; mathematical modeling; EPC50; system simulation; Visual C++

\section{INTRODUCTION}

With the development of the marine shipping industry, maritime colleges and universities came into being. For the purpose of training students, they mainly provide students with training venues. Because the ship oil purifier systems have the particularity of environment and application. In addition, the new colleges and universities is limited by experiment and teaching sites. To sum up, most of the laboratory cannot be arranged in accordance with the actual ship layout plan [1]. The actual ship oil purifier system has complex structure and high cost. So, the actual ship training for crew has great limitations. The economy and the convenience of training crew in the oil purifier simulation system will be hard to realize in the actual ship. Currently, most simulators of ship oil purifier simulation system are given priority to with the hardware and many shortcomings of usage. With the development of computer simulation technology and the improvement of training demands suggested by the international maritime organization for simulation simulators, a complete set of oil purifier simulation system to train students has become a trend. Therefore, it's necessary to design an oil purifier simulation system. It can reflect the actual working condition of the oil purifier accurately, and also simulate the dynamic process and state phenomenon of ship oil purifier realistically. It has a vital role of making the students master the essentials and improving students' ability of analysis and solving problems.

\author{
Zhipeng Shen*, Changsheng Dai and Haiwei Wang \\ School of Information Science and Technology \\ Dalian Maritime University \\ Dalian, China \\ *Corresponding author
}

Foreign turbine simulator is developed earlier and general the level of hardware and software is higher, but the price is more expensive. Norwegian Kongsberg as well as German Siemens whose products in the world have leading level is most famous company in this field. In China, research and development of turbine simulator started relatively late and experimental condition is poor, but with the high starting point, development is also booming. Hua Yan developed ship oil purifier simulation system by PLC control in Jimei University in 2012[2]. The system can control oil purifier effectively, but need high maintenance cost owing to giving priority to with hardware. Jun $\mathrm{Wu}$ designed oil purifier separation model and controller model in Dalian Maritime University in 2009. In .Net development environment, he used Visual C\# programming language to develop fuel oil purification system simulation software [3].But the established mathematical model is simple and accuracy is not high, the interface has many shortcomings of usage. East China normal university in 2010 under the integrated development environment, JunHui Bao used VB programming language to develop interface and established mathematical model for oil purifier simulation system [4]. The control model of reference simulation data and the actual one have certain differences, and software interface is not strong immersive.

The article regarded the oil purifier system in the COSCO PACIFIC thous ands of container ships as the simu lation object. It established dynamic oil purifier separation model, oil water interface model, mathematical model of pipe and pump and oil tank model. With carrying on the thorough analysis to the logic sequential control of EPC50. By using C++ language in $\mathrm{VC}++$ 6.0 programming environment, it realized interface design of the oil purifier system including fuel oil, main sliding oil and auxiliary sliding oil. The system interface can reflect constituent parts of real oil purifier system comprehensively and realistically, and the established mathematical model and logic sequential control simulates truly in strict accordance with the actual oil purifier situation.

\section{OIL PURIFIER SYSTEM MATHEMATICAL MODELING}

The article regarded oil purifier system in the COSCO PACIFIC thous ands of container ships as the simu lation object. The oil purifier system of this kind of ships is produced by ALFALA VAL including three models known as SU881 fuel oil purifier, 2 models known as SU866 main sliding oil purifier 
and 2 models known as PA625 auxiliary sliding oil purifier, and other parts of 7 oil pu mps, oil tanks and valves. The model of oil purifier simulation system is consist of four parts, namely oil purifier dynamic separation model, oil water interface model, Pipeline valve model and oil pump model.

\section{A. Oil Purifier Dynamic Separation Model}

Oil purifier is main ly to make impurities separate from oil, and its basic principle is using centrifugal force produced by high speed rotation to separate larger components such as the impurity particles, water and the elements of larger density in oil purifier between tiny spaces separating plate [5]. When the particles (solid or liquid) are under the action of gravity, it will get a constant speed through the viscous mediu m after a certain time. If following physical parameters has been known, deposition speed can be calculated by deposition rate with the inference of Stokes' theorem:

$$
v_{g}=\frac{d^{2}\left(\rho_{p}-\rho_{1}\right)}{18 \eta} g
$$

If the separation bowl is filled with liquid and make it turning, it will produce a centrifugal field. $a$ - centrifugal acceleration; $\omega$ - the angular velocity; $r$ - the radius of separation cylinder;

The acceleration equation can be expressed as:

$$
a=r \omega^{2}
$$

The acceleration $a$ is plugged in (1) and the speed of separation cylinder is calculated by:

$$
v=\frac{d^{2} \Delta \rho}{18 \eta} r \omega^{2}
$$

$d$ - diameter of particles; $\rho_{p}$ - density of particles; ${ }^{\rho_{1}}$ - the density of continuous phase; g-acceleration of gravity; $v_{g}$ - the rate of gravity sedimentation; $\Delta \rho$ - density contrast ; $\eta_{\text {- the }}$ viscosity of continuous phase; $\omega_{-}$the angular velocity ; $r$ - the radius of separation cylinder; $v$ - the separation rate inside separation cylinder[6]. Particles make laminar motion in the height of $h_{\text {with a speed of }} V_{s}$ in annular space, it can be expressed as:

$$
\begin{gathered}
V_{s}=\frac{1}{2 \delta} * \frac{\Delta \rho}{l}\left(y^{2}-h y\right) \\
\frac{d x}{d y}=\frac{1}{2 \eta} * \frac{\Delta \rho}{l} y^{2}-\frac{1}{2 \eta} * \frac{\Delta \rho h}{l} y+\frac{\Delta \rho d^{2} \omega^{2} r \cos \varphi}{18 \eta}
\end{gathered}
$$

$$
\frac{d y}{d t}=\frac{\Delta \rho d^{2} \omega^{2} r \sin \varphi}{18 \eta}-\frac{\Delta \rho d^{2} \omega^{2} r \sin \varphi \cos \varphi}{18 \eta} x
$$

$l$ - length of the separation channel; $h$ - height of the separation channel; $y$ - height offset of particle trajectory; $x$. horizontal offset of particle trajectory.

\section{B. Oil Water Interface Model}

The principle of oil purifier is separating the impurities and water from oil through centrifugal force produced by high speed rotation. Due to their density differently, the centripetal force is also different. The minimum density Oil has the smallest centripetal force, impurities should be gathered in the inside layer of the separation cylinder. On the contrary, the high-density oil has larger centripetal force, water and impurities gathered in the outer layer of the separation cylinder. Oil and water will form stable interface, when the centrifugal pressure is equal.

$$
\begin{gathered}
P=m \omega^{2} R \\
m_{1} \omega^{2} R_{1}=m_{2} \omega^{2} R_{2}
\end{gathered}
$$

$P_{\text {-separation cylinder pressure; }} m_{1} m_{2}$ - quality of oil and water; $\omega$ - angular velocity of separation cylinder; $R_{1} R_{2}$ radius of gyration between quality center and rotation spindle center.

$$
m=\frac{W}{g}=\frac{(S * e) * \rho}{g}
$$

\section{OIL PURIFIER SEQUENCE CONTROL}

Control unit EPC50 module of oil purifier is shown in the figure 1 , which mainly through logic sequential controlled oil purifier.

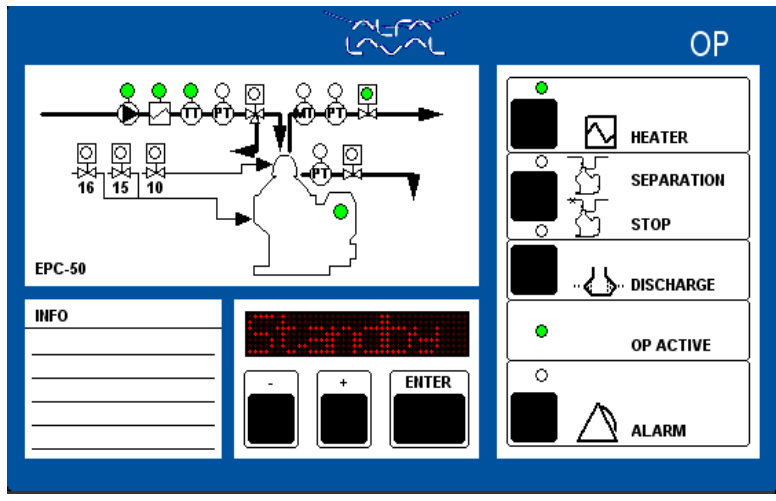

FIGURE I. CONT ROL UNIT OF OIL PURIFIER EPC50 
When valves and pumps are opened entirely, start the oil purifier and press the heating button then wait a while. When the speed of oil purifier and oil temperature reaches the required value, LED displays as 'Standby'. The current state including sequence of time, temperature and pressure can be displayed by pressing the '+' '-' button. Confirm the operation by pressing 'ENTER' button.

Oil purifier enters the startup sequence by pressing key 'SEPARATION' and LED display shows 135 seconds countdown, in the meantime, the oil light shines. After the end of the countdown it enters the separation sequence. After the separation sequence it enters slag discharge sequence automatically or manual mode can be chosen. After that, compensation water enters into oil purifier. This process generates tray of oil purifier falling down and slag discharges from oil purifier to the sludge tank. It turns into normal separation sequence after the slag discharge sequence without receiving the stop-order. On the contrary, it turns into the stop separation sequence with LED showing 'Stop' if the stop-order is taken, and rotational speed decreases to zero, eventually stops separation process. The flow chart of sequential control of oil purifier simulation system is shown in figure 2 .

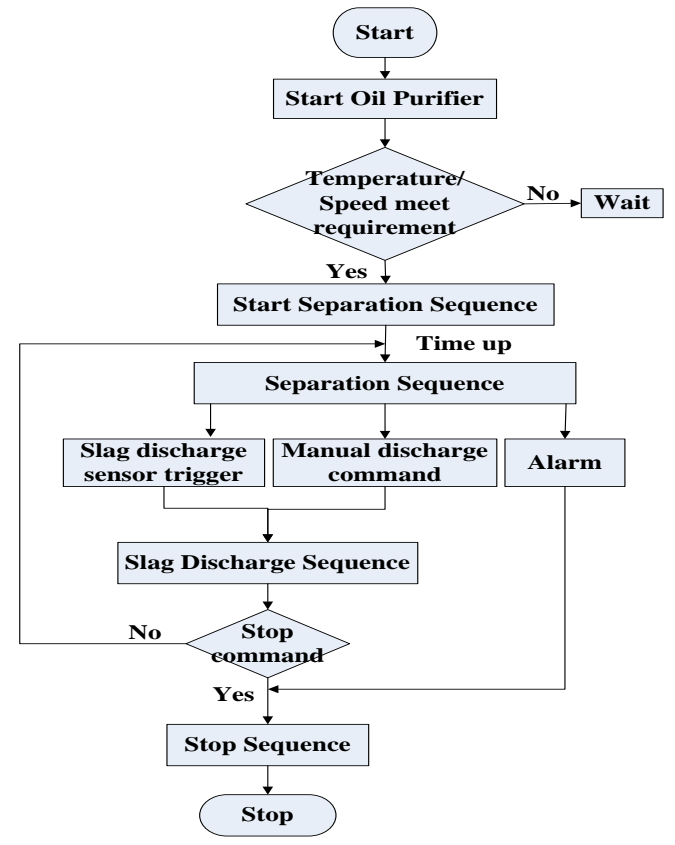

FIGURE II. FLOW CHART OF OILPURIFIER SEQUENCE CONTROL

EPC50 has automatic alarm module. When an alarm occurs, 'ALARM' lamp lights which is upper-left to the control panel is shining and LED display screen displays spots of alarm. Next, the 'ALARM' light is on light after pressing the 'ALARM' button. It'll make the corresponding processing after seeing the alarm content.

\section{A. Startup Sequence}

When a start signal comes, the main program will judge which sequence the oil purifier lies in. If sequence is one of startup, separating or slag discharge sequence, the startup sequence is executed immediately after the stop sequence is finished. After entering the startup sequence, at first closed water solenoid valve V16 should be open, and then water should be supplied for 15 seconds. It'll make the tray of oil purifier rise in order to close the discharge port. After that, the motor should be checked whether the speed achieves rated speed. If reaches the oil temperature should be tested through sensors to find it whether beyond the given value. If it's for sure, the corresponding alarm for high oil temperature and low one works. If Oil temperature is in the given range, feed water solenoid valve V10 should be open to enter water for 60 seconds forming liquid seal. Three-way valve opens automatically after startup sequence starting 75 seconds, then began to oil-taking. Oil purifier starts ingress of oil for another 50 seconds. After the end of the countdown, oil light remains on with the end of the startup sequence and enters into the normal oil separation sequence.

\section{B. Oil Separation Sequence}

After the main program is initialization, at first WT200 should be tested whether can work normally. If not, then alarm immediately. Otherwise, oil pressure should be tested to certain its state. If oil pressure becomes high or low, the corresponding alarm should be taken. If oil pressure is right, then sequence continues to test whether the slag discharge time reaches maximum. If reaches, the displacement water should be injected, then it finished slag discharge once. Otherwise, sequence tests whether reaches the setting value. Then sequence checks whether more than 10 minutes from last slag discharge. In the event of, it returns the main program to slag again. If not, V9 named Drainage solenoid valve is open only 20 seconds. If oil in water is higher than the setting value, V9 is open for 120 s and sequence slag discharge once. If it still higher than the trigger value, then open V9. If up to 120 seconds, it will alarm.

\section{SYSTEM SIMULATION}

Oil purifier simulation system is mainly divided into the fuel oil purifier system, main lube oil purifier system and auxiliary lube oil purifier system. This system creates project based on MFC dynamic link library in $\mathrm{VC}++6.0$ programming environment. Create a new project and then choose the MFC Extension DLL(using shared MFC DLL) in order to create DLL files through MFC AppWizard. In the new project, click the 'ClassView found' and add the corresponding class according to the needs [7]. Choose 'ClassWizard' of the view in the menu bar, add class and select base class. The three systems has a respective class and each one has a corresponding interface, in the system namely 'CFOPSView', 'CMLPSView' and 'CALPSView'. In order to avoid a lot of repetitive tasks, create a class called 'CBaseView' which realizes the dynamic display of pipe, valve, pump and oil tank and set it to the base class of above three classes. In three of the derived classes, directly call the dynamic display functions of four parts in the base class.

\section{A. Fuel Oil Purifier System Interface}

Fuel oil purifier system interface is shown in figure 3, the three fuel oil purifiers of the system are SU881 coming from ALFALA VAL. This type of motor is $35.6^{*} 3600\left(\mathrm{Kw}^{*} \mathrm{Rpm}\right)$ 
and capacity of oil purifier is $9850 \mathrm{~L} / \mathrm{h}\left(\mathrm{AT} 700 \mathrm{cSt} / 50^{\circ} \mathrm{C}\right)$. The two fuel supply pumps of the system are H.GEAR coming from HEISHIN PIMP WORK CO.LTD. This type of motor is $5.5 * 1200\left(\mathrm{Kw}^{*} \mathrm{Rpm}\right)$ and capacity of oil purifier is $8.85^{*} 0.3$ MPa D.P. $(\mathrm{m} 3 / \mathrm{h} * \mathrm{mTH})$ and pump model is M-10B. Three of fuel oil heaters are heating through steam, and valves and pumps are operable. Compared with the other two, there is some special aspects in the No. 1 oil purifier that can choose heavy oil or light oil completing the purification of the heavy oil or light oil[8].

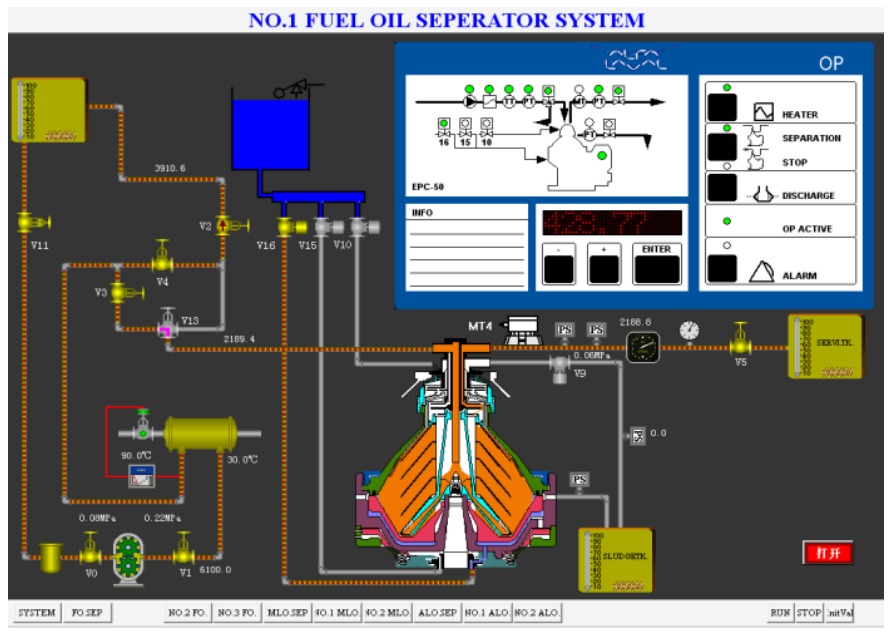

FIGURE III. NO.1 FUEL OIL PURIFIER INTERFACE

\section{B. Oil Purifier Operation Interface}

There are seven oil purifiers in the three subsystems of oil purifier simulation system, and each one has its own interface. It can ju mp to the corresponding oil purifier operation interface by double-clicking the oil purifier of each subsystem interface. Due to this system being associated with fuel system, lube oil purifier system and power station system, some valves and pumps cannot be operated in the interface and need to change them in their corresponding system, but the state of the pump and valve can be displayed. Each system has the same oil purifier operation interface, so No.1 fuel oil purifier shown in figure 3 can be taken as an example to represent others. The pop-up 'start or stop' dialog is shown after clicking the 'open' button in bottom right corner.The pointer of ammeter waves violently after starting oil purifier and it won't stabilize at a certain value until the rotate speed and temperature meet some require ments.

\section{1) Oil purifier startup operating manually}

a. Check whether the power of the oil purifier is normal, whether the oil level of oil pan is normal and whether in and out of the line of the valves are in the normal position.

b. If the above conditions are normal, then start the supply pump of the oil purifier and open the steam heating value. Start the heater from the operation panel. c. After the temperature meets the requirements, press the SEPARATION/STOP(SEPARATION) button to activate the controller. The LED window will scroll appear a few problems, we must answer these questions first, then be allowed to start the equipment. Start oil purifier, wait the speed of oil purifier at full speed and oil temperature over low-alarm-value temperature, control system will automatically enter the startup sequence until enter normal separation sequence.

d. Observe the oil purifier after supplying oil normally, whether the abnormal noise or vibration exist. Judge the condition of purifier through the data displayed on the controller panel. If the condition is normal, it means the oil purifier in normal situation.

\section{CONCLUSION}

This oil purifier simulation system with dynamic humancomputer interaction interface can simulate the real operation process and display the running state of the oil purifier visually. It also can adjust the running parameters to operate oil purifier and set all kinds of fault alarm to judge the operation state. Sequence in the simulation system is same as the one in the real ship and the simulated results reflect the actual dynamic process of system. Above-mentioned description proved the logic analysis is reasonable and the established mathematical model is accurate and simulation tool is appropriate. Oil purifier simulation system can make the students to master the working principle of oil purifier fully. It plays an important role to make the students enhance the intuitive understanding of oil purifier system and improve their abilities to analyze and solve problem and gain valuable experiences.

\section{ACKNOWLEDGMENT}

This work is partially supported by the Fundamental Research Funds for the Central Universities (3132015106, 3132015040).

\section{REFERENCES}

[1] Yuting Wang. Research on the Virtual Operational System for Marine Auxiliary Boiler. Dalian: Dalian Maritime University, 2012.

[2] Hua Yan. The Development of Marine Oil Separator Operation Training Sinmulation. Mechanical and Electrical Technology, 2012, pp. 42-44.

[3] Junhui Bao. Design and ImPlementation of Simulation System for Marine Oil Separat or Control. ShangHai: East China Normal University, 2010.

[4] Jun Hu. Design and Research on Marine Fuel Oil Purifying System. Dalian: Dalian Maritime University, 2009.

[5] Shichen Li, Huibing Gan, Benteng Zou, Jundong Zhang. Design and Realization of Marine Purifier Simulation System. Navigation of China, 2009, pp. 43-47.

[6] Heng Wu, Haoji Li. Alfa Laval Modern Technology Separator Section. Dalian: Dalian Maritime Publishing Company, 1995.

[7] Xin Sun. VC++ in-depth Xiangie. Beijing: Electronic Industry Publishing Company, 2012.

[8] Qiang Ma, Hongyan Mao, Baozhu Jia. Purifier System Simulation Design. Navigation of China, 2010, pp. 46-49. 